\title{
Higher-order Structure of Human Chromosomes Observed by Electron Tomography and Electron Diffraction
}

Misa Hayashida ${ }^{1}$, Rinyaporn Phengchat ${ }^{2}$, Darren Homeniuk ${ }^{3}$, Marek Malac ${ }^{4}$, Ken Harada ${ }^{5}$, Tetsuya Akashi $^{6}$, Nobuko Ohmido ${ }^{2}$ and Kiichi Fukui ${ }^{7}$

${ }^{1}$ National Research Council Canada, Edmonton, Alberta, Canada, ${ }^{2}$ Kobe University, Kobe, Hyogo, Japan, ${ }^{3}$ NRC-NANO, Edmonton, Alberta, Canada, ${ }^{4}$ NRC-NANO \& Physics, U of Alberta, Edmonton, Alberta, Canada, ${ }^{5}$ RIKEN, Hatoyama, Saitama, Japan, ${ }^{6}$ Hitachi, Ltd., Hatoyama, Saitama, Japan, ${ }^{7}$ Osaka University, Suita, Osaka, Japan

Chromosome higher-order structure has been an enigma for over a century. Proposed models include radial loop model, radial coil model, folded fiber model and helical coiling of radial loops model [1]. These models are based on the assumption that DNA is wrapped around histones and forming nucleosomes, and nucleosome fiber with $11 \mathrm{~nm}$ is folded into a 30nm basic chromatin fiber. The $30 \mathrm{~nm}$ chromatin fibers in chromosomes have been observed by SEM, TEM and TEM tomography. Several results also support existence of $30 \mathrm{~nm}$ chromatin fiber structure [2,3]. On the contrary, the $30 \mathrm{~nm}$ chromatin structures in mitotic chromosomes were not found by cryo-EM [2,3] and small-angle X-ray scattering results. Larger 200-400 nm structures consisted of the $30 \mathrm{~nm}$ fibers have also been observed although the structures have not been supported by several results.

Some models such as radial loop model support existence of axially-positioned chromosome structure, termed as 'chromosome scaffold'. The chromosome scaffold is thought to be as a key structure of mitotic chromosome. It is considered that the scaffold plays a vital role in chromosome condensation, shaping Xshaped structure of mitotic chromosome, and also provides flexibility for chromosome movement during cell division. However, some models such as folded fiber model suggest that there is no axially structure in chromosomes. The reason of these inconsistencies might have come from artifacts caused in the sample preparation.

Here, we prepared chromosomes for electron tomography observation with ionic liquid instead of conventional dehydration which is likely a source of artifacts. Ionic liquids essentially coat and penetrate into the sample, preventing the sample from drying by replacing water with it under vacuum conditions $[4,5]$. To observe 3D structure of scaffold and DNA fibers, two types of chromosome samples on Carbon/Fomvar support film on TEM grid were prepared as follows.

(A) Chromosomes with immunogold-labeled condensin complex, one of the major chromosome scaffold proteins [6]

(B) Chromosomes stained using ChromEM, a DNA-labeling method developed for electron microscope observation $[4,7]$ and osmium.

Both samples were treated with ionic liquid [8]. For electron tomography, a chromosome from each sample was picked from the Carbon/Fomvar support film and transferred to a $360^{\circ}$ rotation holder $[9,10]$ using the method shown in Fig. 1 in HITACH NB5000 FIB/SEM to avoid a missing wedge effect [6]. The beam current of FIB was $0.01 \mathrm{nA}$, which is $10 \mathrm{x}$-to $100 \mathrm{x}$ lower than normal FIB fabrication. To avoid radiation damage by FIB, the area of interest was scanned by FIB only a few times for rough adjustment 
of the probe position. Otherwise, the position of the probe and the area of interest were adjusted precisely using SEM images.

HITACH HF3300 with $300 \mathrm{kV}$ acceleration voltage and Hitachi H-1000FT with $1 \mathrm{MeV}$ acceleration voltage were used to take tilt series of sample (A) and (B), respectively.

Fig. 2 is a tomogram of sample (A) from two directions after removing diffusion of gold nanoparticles around the scaffolds. The tomograms of gold nanoparticles inside chromosomes showed similar helical patterns to chromosome scaffold organization reported based on observations with 3D SIM and FIB/SEM [11]. The pitch is approximately $200 \mathrm{~nm}$. In the tomogram of sample (B), $200 \mathrm{~nm}$ periodicity perpendicular to chromosome axis was also observed. The results from sample (A) and (B) indicates both structures of scaffold and DNA fibers are related and scaffold plays a vital role in chromosome organization.
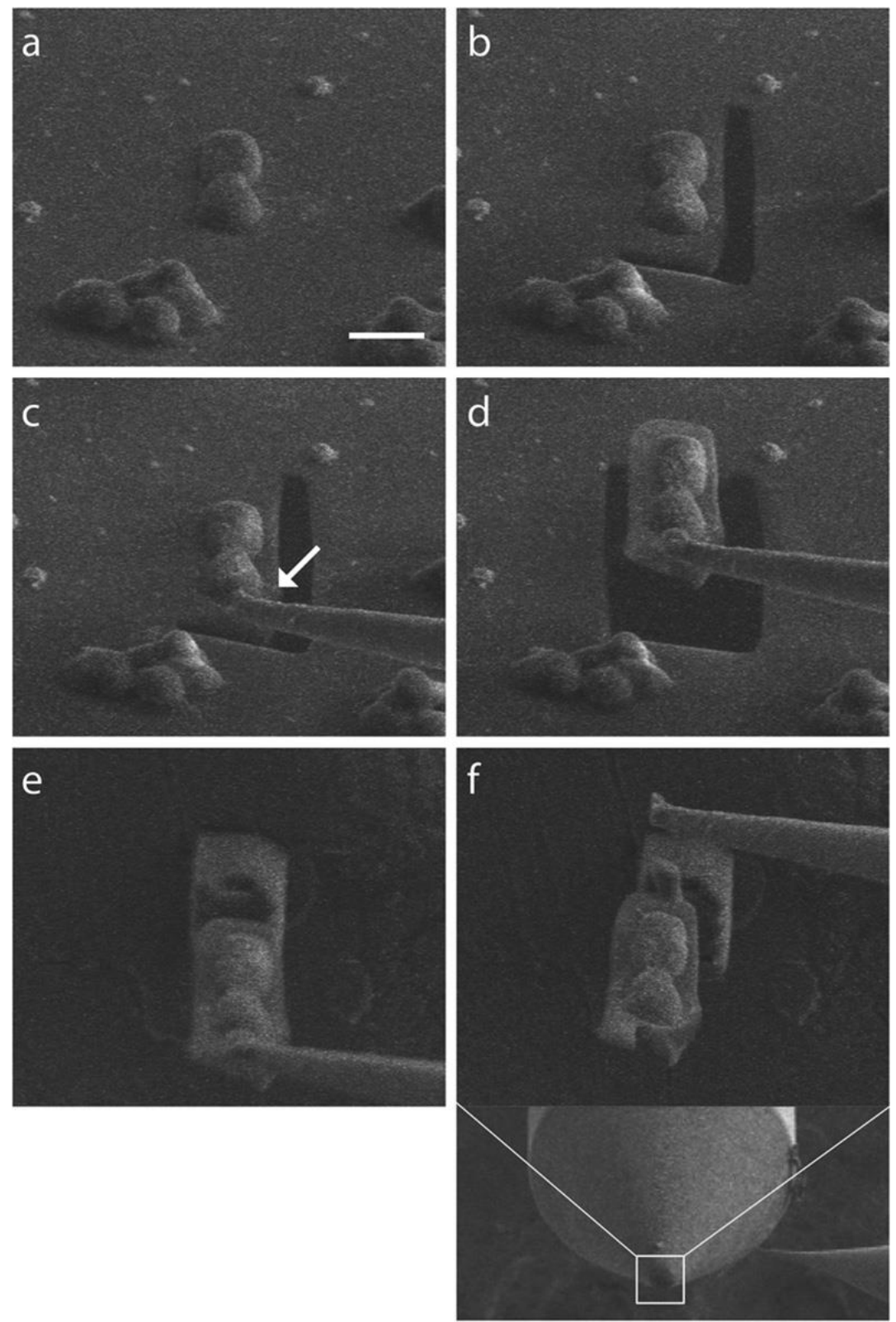
Figure 1. Schematic procedure images of transferring a chromosome to a $360^{\circ}$ electron tomography holder a) Area with a selected chromosome b) Right and bottom sides of the chromosome were cut with FIB. c) A micro-sampling probe was extended and attached to the area using carbon deposition. d) Remaining sides of the area were cut by FIB and the area was released from the Formvar/Carbon film. e) The area was attached to a carbon platform on a $360^{\circ} \mathrm{ET}$ holder using carbon deposition. f) The microsampling probe was detached with FIB while the chromosome remained attached to the holder. An inset in $\mathrm{f}$ ) showed the position of a chromosome on the $360^{\circ}$ holder at low magnification. These images were taken in SEM mode. Scale bar=2 $\mu \mathrm{m}$. [6] Copyright 2019 Elsevier.

a

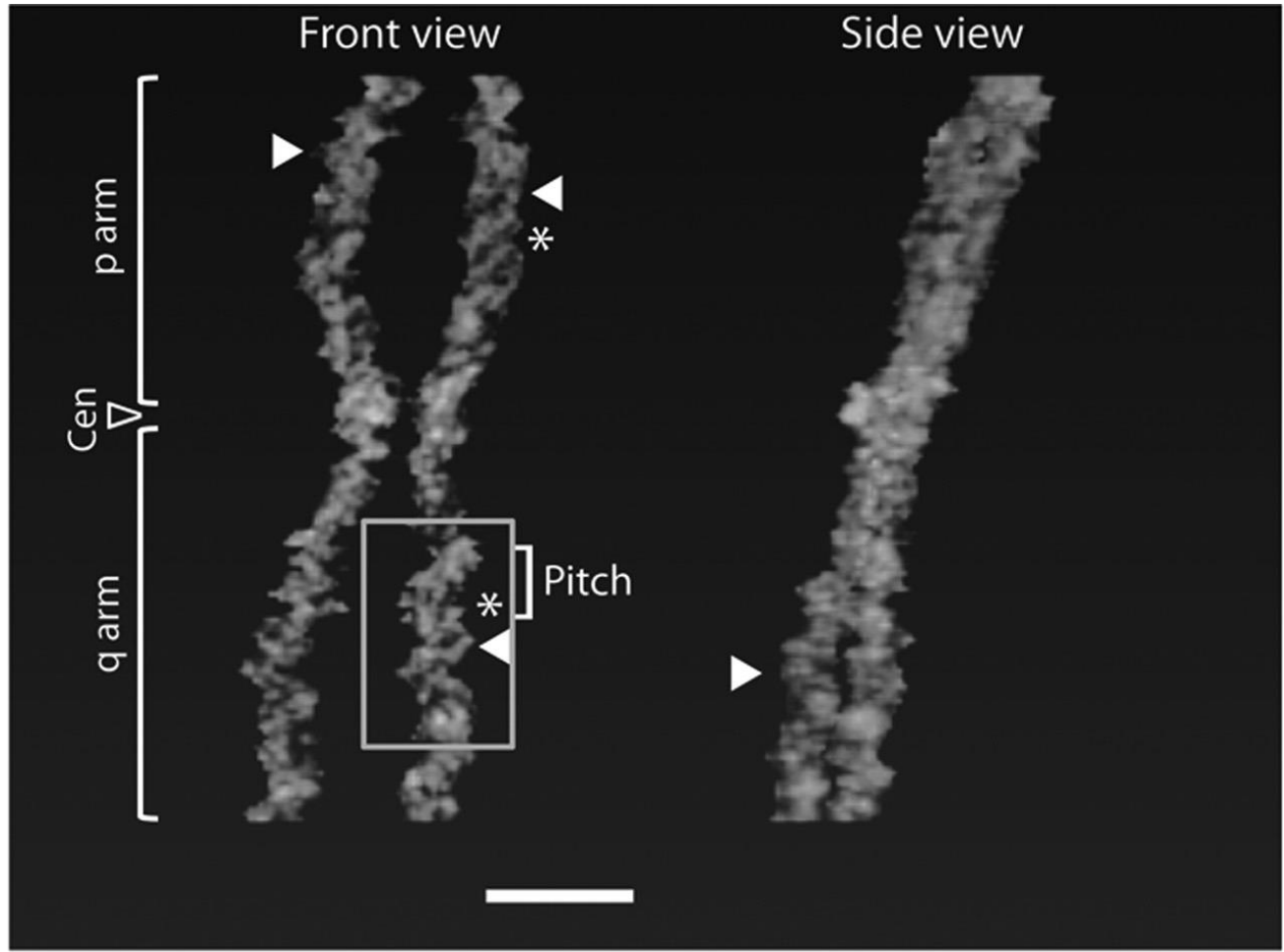

b

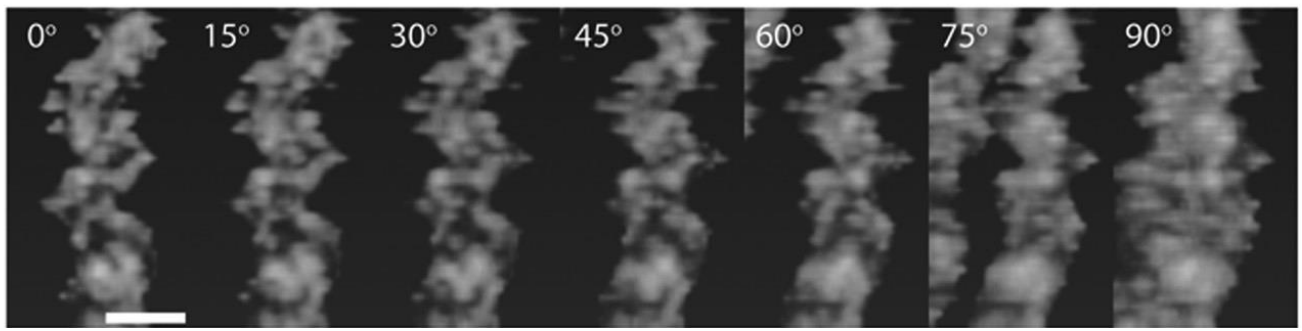

Figure 2. A tomogram of chromosome scaffold having a helical structure a) A tomogram of a chromosome scaffold b) Images showing magnified view of a box in (a) with $0-90^{\circ}$ tilt range. [6] Copyright 2019 Elsevier.

\section{References}

[1] T. Ushiki, O. Hoshi, K.I. Iwai, E. Kimura, M. Shigeno, Archives of Histology and Cytology, 65 (2002) 377-390.

[2] K. Maeshima, M. Eltsov, J Biochem, 143 (2008) 145-153.

[3] K. Maeshima, S. Hihara, H. Takata, 75 (2010) 439-444.

[4] T. Welton, Chemical Reviews, 99 (1999) 2071-2083.

[5] S. Kuwabata, A. Kongkanand, D. Oyamatsu, T. Torimoto, Chemistry Letters, 35 (2006) 600-601. 
[6] R. Phengchat, M. Hayashida, N. Ohmido, D. Homeniuk, K. Fukui, Micron, 126 (2019) 102736.

[7] H.D. Ou, S. Phan, T.J. Deerinck, A. Thor, M.H. Ellisman, C.C. O'Shea, Science, 357 (2017).

[8] A. Dwiranti, L. Lin, E. Mochizuki, S. Kuwabata, A. Takaoka, S. Uchiyama, K. Fukui, Microsc Res Tech, 75 (2012) 1113-1118.

[9] R. Tsuneta, H. Kashima, T. Iwane, K. Harada, M. Koguchi, Microscopy (Oxf), 63 (2014) 469-473. [10] T. Yaguchi, M. Konno, T. Kamino, M. Watanabe, Ultramicroscopy, 108 (2008) 1603-1615.

[11] R. Poonperm, H. Takata, T. Hamano, A. Matsuda, S. Uchiyama, Y. Hiraoka, K. Fukui, Sci Rep, 5 (2015) 11916. 